\title{
Effect of the Silanization Processes on the Properties of Oxidized Multiwalled Carbon Nanotubes
}

\author{
B. Scheibe*, E. Borowiak-Palen and R.J. Kalenczuk \\ Centre of Knowledge Based Nanomaterials and Technologies, Institute of Chemical and Enviroment Engineering \\ West Pomeranian University of Technology, ul. Pułaskiego 10, 70-322 Szczecin, Poland
}

\begin{abstract}
This paper presents the results of the research on the effect of the two different silanization methods on the thermal and structural properties of oxidized multiwalled carbon nanotubes. As-purified material was oxidized in the mixture of nitric and sulfuric acids. An oxidized material was divided into two parts which underwent two silanization treatments by 3-aminopropyltriethoxysilane (APTES). The first experiment (I) was performed at room temperature in acetone $(\mathrm{pH} 7)$ for $30 \mathrm{~min}$. The second experiment (II) involved a hydrolysis of APTES at $40^{\circ} \mathrm{C}$ in water $(\mathrm{pH} 4)$ for $3 \mathrm{~h}$. The functionalization extent of the samples at each step of the preparation was investigated by the Raman spectroscopy. The presence of the functional groups on the nanotubes surface have been studied via the Fourier transform infrared spectroscopy (FT-IR). The quantity of the external moieties introduced on multiwalled carbon nanotubes surface after oxidation process was estimated by the Boehms titration method. The high resolution transmission electron microscopy analysis allowed us to observe the changes of the morphology of the investigated carbon nanotubes. The influence of the silanization processes on the thermal stability of multiwalled carbon nanotubes was thoroughly studied by thermogravimetry analysis. There was observed a significant increase of the thermal stability of the multiwalled carbon nanotubes samples upon silanization treatment in respect of pristine and oxidized multiwalled carbon nanotubes.
\end{abstract}

PACS numbers: 81.65.Mq, 81.07.De, 61.48.De, 65.80.--g, 68.37.Lp

\section{Introduction}

Since their discover the multiwalled carbon nanotubes (MWCNTs) have attracted attention of the researches from many different branches of science due to their outstanding properties such as wide surface area, chemical and thermal stability, ultra-light weigh, high tensile strength and electronic properties [1-4]. Utilization of MWCNTs features can be broadened by an egzohedral functionalization. The modification process can involve single or several steps. The single step functionalization of carbon nanotubes by oxidation process usually leads to an introduction of the linkers for more sophisticated functionalities [5-7]. The introduction of the functional groups on MWCNTs surface provides the active sites for binding other organic/inorganic molecules such as organosilanes, proteins, polymers [8-10]. The hydroxyl groups introduced via oxidation process onto MWCNTs outer walls are very attractive for the silanization process where they act as the anchoring points $[4,11,12]$. The binding of the 3-aminopropyltriethoxysilane (APTES) molecules to the inorganic hydroxylated surface is an effective method for the modification of the physical and chemical properties of the treated material $[4,13,14]$. The silanization process enables binding of carbon nan-

* corresponding author; e-mail: bscheibe@zut.edu.pl otubes to guest molecules such as proteins, polymers or drugs via amide bonds. Therefore, silanized carbon nanotubes can be potentially applied in fields of polymer nanocomposites, biosensors, and drug delivery systems $[10,15,16]$. So far aminosilane grafting technique was used to improve the solubility of CNTs, reinforced polymer nanocomposites, or in biosensor design [15]. The modification of the MWCNTs egzohedral surface via silanization process is a complex process due to the reactivity of the aminosilane molecules with inorganic surface which depends on the reaction time, $\mathrm{pH}$ and nature of the solvent, temperature and silane concentration [13]. The influence of the specified parameters on the silanization process is related to the chemical structure of APTES molecule being composed of hydrolytically stable bond $\mathrm{H}_{2} \mathrm{~N}-\left(\mathrm{CH}_{2}\right)_{3}-\mathrm{Si}$ and a second part: $\mathrm{Si}-\left(\mathrm{OC}_{2} \mathrm{H}_{5}\right)_{3}$ which can easily undergo the hydrolysis process forming trisilanol [13].

In the present study, two silanization procedures were used to modify multiwalled carbon nanotubes surface with APTES molecules. In the first experiment (I) the silanization proces occurs in one step. The APTES molecules were mixed with MWCNTs dispersed in acetone at room temperature. The grafting of the aminosilane molecules onto MWCNTs surface was the result of the $\mathrm{Si}-\left(\mathrm{OC}_{2} \mathrm{H}_{5}\right)_{3}$ bond breaking in the presence of hydroxyl functional groups [12]. In the second experiment (II) the silanization occurred in two steps. In the 
first step, MWCNTs were dispersed in acid solution at $40{ }^{\circ} \mathrm{C}$ which resulted in a hydrolysis of the ethoxy groups $\left(\mathrm{OC}_{2} \mathrm{H}_{5}\right)$ which were replaced by hydroxyl groups $(\mathrm{OH})$ forming reactive trisilanol. A self-condensation of the trisilanols produced siloxane bonds ( $\mathrm{Si}-\mathrm{O}-\mathrm{Si}$ ) forming aminopropyl-functional resinous oligomers and polymers. In the second step, the polymer was associated with the multiwalled carbon nanotubes surface and formed covalent bonds in defect sites containing $\mathrm{OH}$ groups [13]. The detailed spectroscopic, microscopic and thermogravimetric analysis of the prepared samples have been performed. Interestingly, it was observed that along with the degree of the aminosilane functionalization, the thermal stability of the modified MWCNTs samples in respect of the pristine and oxidized MWCNTs significantly increased.

\section{Experimental}

\subsection{Materials}

APTES 99\% was obtained from Aldrich. Sulfuric acid $96 \%$ p.a. was purchased from Cheman. Chloric acid 35-38\% p.a., nitric acid $65 \%$ p.a., acetone $99.5 \%$ p.a. were purchased from Chempur.

\subsection{Synthesis, purification and oxidation of MWCNTs}

Multiwalled carbon nanotubes were synthesized via chemical vapor deposition (CVD) process [17]. The purification procedure involved chloric acid treatment in ultra-sonication bath for $3 \mathrm{~h}$ and subsequent multiple filtration through the polycarbonate filter (Whatman pore size $0.2 \mu \mathrm{m}$ ) in order to remove the majority of the dissolved metal particles. Next, carbon nanotubes were dried under vacuum conditions at $180^{\circ} \mathrm{C}$ for $1 \mathrm{~h}$. The pristine material (P-MWCNTs) was oxidized by the acid treatment in the mixture of $\mathrm{HNO}_{3} / \mathrm{H}_{2} \mathrm{SO}_{4}(\mathrm{v} / \mathrm{v}=1: 3)$ at $175{ }^{\circ} \mathrm{C}$ for $18 \mathrm{~h}$. The acidic MWCNTs solution was diluted with water purified in reversed osmosis process $\left(\mathrm{RO} \mathrm{H}_{2} \mathrm{O}\right)$ and filtrated through the polycarbonate filter. Then, the sample was rinsed with $\mathrm{RO} \mathrm{H}_{2} \mathrm{O}$ and acetone. Then, oxidized material (O-MWCNTs) was dried under vacuum conditions at $180^{\circ} \mathrm{C}$ for $1 \mathrm{~h}$ in order to degas adsorbed $\mathrm{CO}_{2}$ and $\mathrm{H}_{2} \mathrm{O}$.

\subsection{Silanization of MWCNTs surface}

The $10 \mathrm{mg}$ of previously oxidized MWCNTs were divided into two parts and mixed with $10 \mathrm{ml}$ of $\mathrm{RO} \mathrm{H}_{2} \mathrm{O}$ in the test tubes (Falcon - $50 \mathrm{ml}$ ) connected to the vacuum pump. The O-MWCNTs solutions were dispersed under vacuum conditions in ultra-sonication bath at $60{ }^{\circ} \mathrm{C}$ for $20 \mathrm{~min}$. After that treatment the oxidized MWCNTs underwent two different processes leading to the silanization. In the experiment I the O-MWCNTs solution was gently filtrated through the polycarbonate filter and rinsed thoroughly with acetone in order to remove adsorbed water molecules which could promote hydrolysis process. Next, the obtained sample was re-dispersed in aceton in the test tube and placed onto the magnetic stirrer. Then, APTES solution was added slowly to the dispersed O-MWCNTs solution until its concentration of $2 \%$ was reached. Subsequently, the mixture was stirred under vacuum conditions for $15 \mathrm{~min}$. Next, the pressure was gently elevated untill the atmospheric pressure was reached and the mixture was left for $30 \mathrm{~min}$ in closed tube at room temperature. The final product obtained in the experiment I procedure was well dispersed solution. It will be named SI-MWCNTs. In the experiment II the O-MWCNTs solution was also gently filtrated through the polycarbonate filter and rinsed with $\mathrm{RO} \mathrm{H}_{2} \mathrm{O}$. The obtained sample was re-dispersed in $\mathrm{RO}$ $\mathrm{H}_{2} \mathrm{O}$ in the test tube in the ultra-sonication bath under vaccum conditions at $40^{\circ} \mathrm{C}$ for $20 \mathrm{~min}$. Then $\mathrm{pH}$ of the O-MWCNTs solution was adjusted to 4.0 with known amount of glacial acetic acid. Then as previously, APTES solution was added slowly to the dispersed O-MWCNTs solution until its concentration of $2 \%$ was reached. Subsequently, the mixture was sonicated under vacuum condition for $15 \mathrm{~min}$. When the atmospheric pressure was gently recovered, the mixture was left for $3 \mathrm{~h}$ in the closed tube at $40^{\circ} \mathrm{C}$. The final product obtained in the experiment II was weakly solubilized, burdened silanized MWCNTs and will be named SII-MWCNTs. In the next step after each silanization procedure, the mixtures were filtered through a polycarbonate filter, rinsed thoroughly with acetone in order to remove excess of the aminosilane molecules and dried.

\subsection{Characterization of MWCNT samples}

The Raman investigation of vibrational properties was carried out using a micro Raman Renishaw spectrometer $(\lambda=785 \mathrm{~nm})$. For the surface functional group analysis by the Fourier transform IR (FT-IR) spectroscopy [Nicolet 6700 FT-IR Spectrometer], the MWCNT samples were prepared as $\mathrm{KBr}$ pellets. Modified titration method proposed by Boehm was used to estimate the number of oxygenated surface groups. Thermogravimetric analysis was performed on $5 \mathrm{mg}$ samples on DTA-Q600 SDT TA Instruments apparatus with the heating rate of $10^{\circ} \mathrm{C}$ per min from room temperature to $900^{\circ} \mathrm{C}$ in air. The morfological changes of MWCNTs after functionalization and silanization processes were observed via a high-resolution transmission electron microscope (HRTEM) [FEI Tecnai F30].

\section{Results and discussion}

The TEM investigation of the pristine and functionalized samples is depicted in Fig. 1. Figure 1A reveals the morphology of the individual pristine multiwalled carbon nanotube. The pristine material is featured by the characteristic, well-organized cylindric graphene layers. A detailed microscopic analysis proved that almost no amorphous carbon was detected in all the observed sample areas. The microscopic analysis of the functionalized MWCNTs revealed the morphological changes in each investigated sample in respect of the pristine sample. The oxidized and silanized carbon nanotube samples exhibited some additional features on its outer walls. In the 
case of oxidized sample (Fig. 1B) this can be attributed to the defect creation and introduction of the functional groups. However, the silanized samples (Fig. 1C, D) exhibit that the MWCNTs surface is coated by the guest molecules. Additionally, from Fig. 1D in respect of Fig. 1C, one can observe the thicker aminosilane layer which was produced due to APTES hydrolysis and its self-condensation which occurred during the performance of the experiment II.

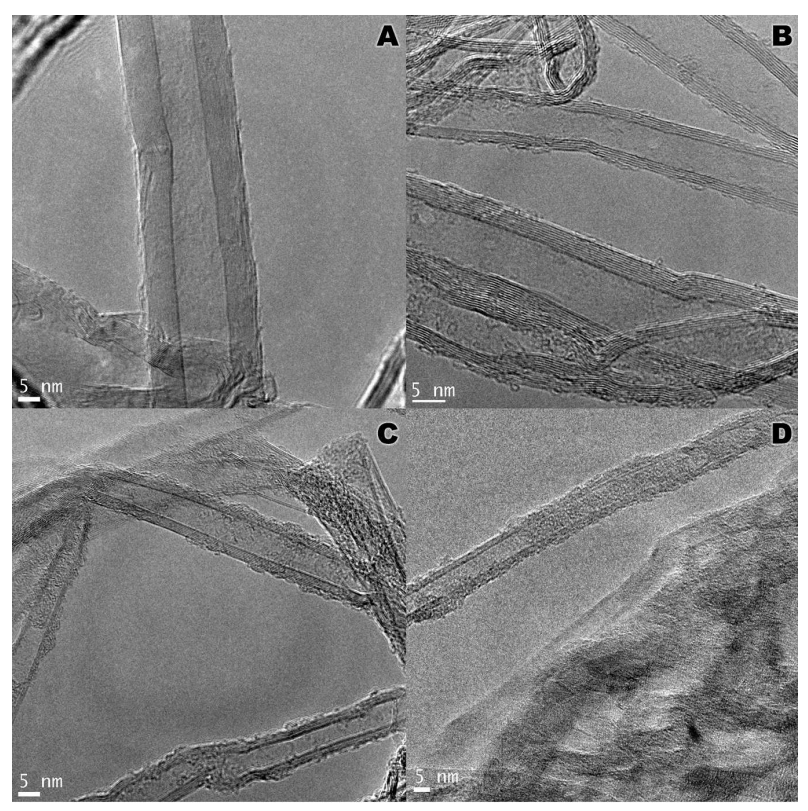

Fig. 1. TEM micrographs of P-MWCNTs (A), O-MWCNTs (B), SI-MWCNTs (C), SII-MWCNTs (D).

The vibrational properties of the pristine, oxidized and both silanized MWCNTs samples were analyzed by the Raman spectroscopy. The Raman spectra of MWCNTs displays the $D$ and $G$ bands. The intensity of $D$-band is proportional to the degree of the structure defects or surface functionalization [18]. These specified effects allow the inelastic scattering needed for the single-phonon, double, or triple resonance effects [19]. Therefore, changes in vibrational properties of P-MWCNTs upon the functionalization via acid treatment and subsequent surface silanizations were estimated via calculation of $I_{G} / I_{D}$ ratio $[20,21]$. From the results presented by the Raman spectra of the investigated samples (data not shown here) it was observed that P-MWCNTs sample was featured by the highest $I_{G} / I_{D}$ ratio $(0.71)$ which indicates good quality of the sample after the purification process. The MWCNTs oxidation process via acid treatment leads to the decrease of the $I_{G} / I_{D}$ ratio $(0.65)$ due to the destruction and creation of the defect sites according to the proposed mechanism of defect-consuming and defect-generation steps [22]. Additionally, the refluxing process introduced oxygen containing surface moieties such as carbonyl, carboxyl, lactone, phenolic and hydroxyl groups. The hydroxyl groups were acting as the anchoring points for the aminosilane molecules during the subsequent functionalization process. The vibrational properties of the both silanized MWCNTs had been significantly modified. The consecutive decrease of the $I_{G} / I_{D}$ ratios of SI-MWCNTs (0.625) and SII-MWCNTs (0.59) samples after the oxidation procces was closely related to the amount of the bonded aminosilane molecules onto MWCNTs surface. From the difference in $I_{G} / I_{D}$ ratio between both silanized samples it can be clearly seen that the double step procedure of the silanization in the experiment II provided a higher functionalization degree of the investigated sample.

FT-IR spectroscopy is a common technique for the identification of the functionalities on the surface of MWCNTs. The vibrational responses of the samples present in Fig. 2 expose the changes in the functional groups content of the pristine multiwalled carbon nanotubes (P-MWCNTs), upon the acid treatment (O-MWCNTs), and after the silanization processes (SI-MWCNTs, SII-MWCNT). In Fig. 2 one can clearly notice the changes in the intensities of the $\mathrm{C}=\mathrm{C}$ absorption band from sample to sample. The intensity increase of the $\mathrm{C}=\mathrm{C}$ vibrational response in O-MWCNTs sample in respect of P-MWCNTs sample could be caused by the introduction of defects and/or functional groups. However, the gradual decrease of the $\mathrm{C}=\mathrm{C}$ band intensities in the silanized samples (SI-MWCNTs, SII-MWCNT) could be due to the aminosilane grafting on the egzohedral wall surface which can be strongly seen in the SII-MWCNTs spectrum. Additionally, the FT-IR spectra of the SI-MWCNTs confirmed the grafting of the aminosilane molecules onto MWCNTs surface by the presence of the established $\mathrm{Si}-\mathrm{O}-\mathrm{C}$ bonds at $1110 \mathrm{~cm}^{-1}$ and $\mathrm{Si}-\mathrm{OH}$ stretch mode at $875 \mathrm{~cm}^{-1}$, respectively. The intensity of those bands increased in SII-MWCNTs which is in agreement with the increase of the APTES egzohedral layer thickness around the tubes observed in TEM. Moreover, in the spectra of SII-MWCNT one can clearly see the presence of the $\mathrm{Si}-\mathrm{O}-\mathrm{Si}$ bonds at $1161 \mathrm{~cm}^{-1}$ which confirms the occurrence of the condensation process.

The amount of the functional groups content was estimated by the Boehm titration method [23]. The quantitative composition of the investigated functional groups onto oxidized MWCNTs surface $(\% w / w)$ was the following: $20.83 \%$ carboxyl, $61.67 \%$ lactone, $8.33 \%$ phenolic hydroksyl, and $9.17 \%$ hydroxyl groups. The low content of the hydroxyl groups which readily react with aminosilane molecules determined the amount of bonded APTES. An increased content of the hydroxyl groups would probably provide more anchoring points and therefore would increase the efficiency of the silanization process. In order to obtain highly hydroxylated MWCNTs material, either conditions of the oxidation process should be changed or the majority of the remaining functional groups should be converted into hydroxyl groups. This issue is currently under investigation.

Thermogravimetric analysis (TGA) of the pristine, oxidized and silanized samples presents their thermal sta- 


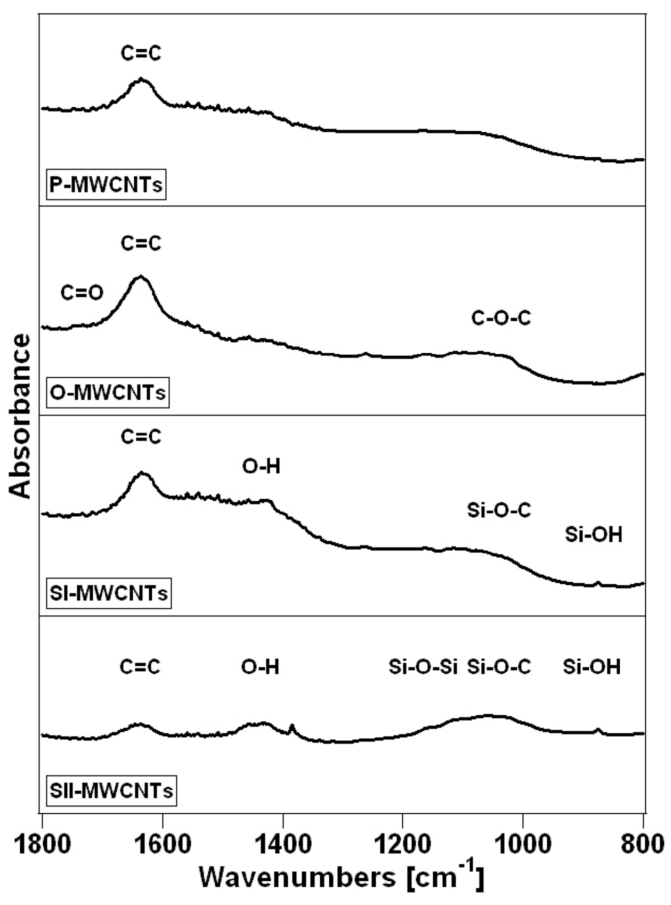

Fig. 2. FT-IR spectra of the MWCNT samples after each step of the preparation.

bility (Fig. 3). It was estimated via the the weight change during the heating process.

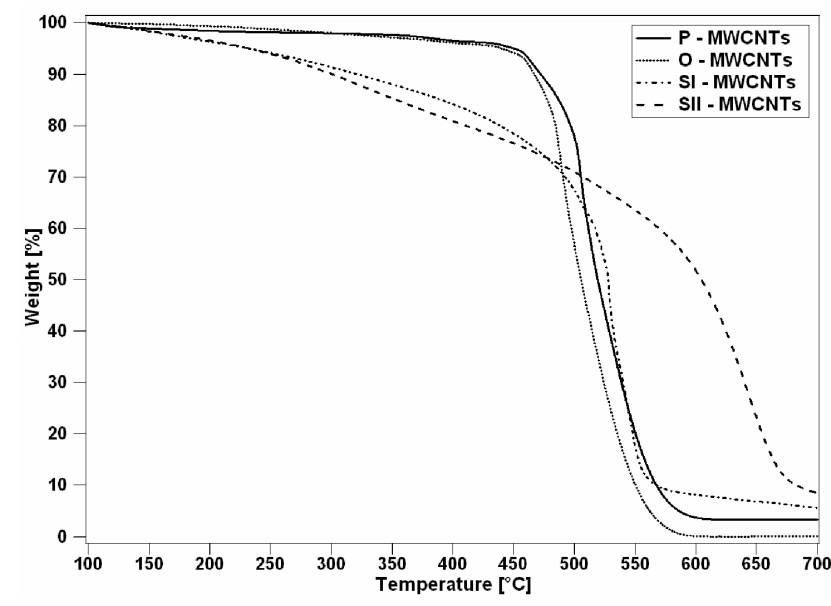

Fig. 3. TG curves of the MWCNT samples after each step of the preparation.

The bold, dot, dash-dot and dash lines presented in Fig. 3 correspond to the TG curves of the pristine, oxidized, and both silanized samples, respectively. TG curve of the pristine sample indicates that after the thermogravimetric process only $3.46 \mathrm{wt} \%$ of the solid material is left. The obtained ashes can be attributed to the remaining catalyst particles. TG curve of the oxidized sample is nearly free $(0.1 \mathrm{wt} . \%)$ of the ashes which indicates that amorphous carbon and remaining cata- lysts were removed during $\mathrm{HNO}_{3} / \mathrm{H}_{2} \mathrm{SO}_{4}$ acid treatment. TG curves of the silanized samples indicate that remaining ashes in the amount of 3.13 wt.\% and 5.89 wt.\% for SI-MWCNTs and SII-MWCNTs originated from the aminosilane molecules. The increased amount of the ashes is closely related to the functionalization degree of the silanized samples. Therefore, a thicker coating around MWCNTs attributed to the aminosilane layer (observed in TEM) obtained in the experiment II left more remaining silicon particles after thermogravimetric analysis. From this figure it can be also observed that initial burning temperature of the functionalized samples decreased along with the increase of the surface functionalization extent. In the case of the oxidized sample the decrease of the initial burning temperature can be due to the introduction of the functional groups and defects upon the acids treatment. However, the consecutive decrease of the initial burning temperature in both silanized samples is related to the introduction of the aminosilane molecules onto MWCNTs surface.

Differential TG (DTG) curves of each TGA data were fitted to the Voigt distribution. Every peak (from 1 to 4 ) corresponding to the stepwise weight loss in DTG profile was carefully studied and the calculations (based on area under the corresponding peak in respect of the sum of all the area peaks) of their fractions in $\%$ are presented in Table.

Low value of the fraction 1 corresponding to the burning off of the amorphous carbon indicates very low content of this component in the pristine multiwalled carbon nanotubes. The temperatures of the remaining fractions (2-4) of these sample correspond to the burning off temperatures of the carbon nanotube material. According to the data obtained from DTG curve of O-MWCNTs one can notice that the absence of the peak corresponding to the amorphous carbon content confirms its efficient removal during the oxidation process via the acid treatment. The three stepwise weight-losses presented by the fractions 2-4 of the oxidized sample correspond to the burning off temperatures of the MWCNTs fractions with different content of the introduced defects/functional groups. The analysis of the DTG curve of the SI-MWCNTs sample exhibits four stepwise weight losses. An oxidation temperature of the fraction 1 corresponds to the oxidation temperature of the weakly bonded APTES molecules, while oxidation temperature of the fractions 2-4 correspond to temperatures of the functionalized material. Fraction 1 in SII-MWCNTs sample was featured by three small, closely positioned peaks which correspond to the weakly condensed aminosilane molecules. The presence of the two consecutive weight losses in fraction 2 seemed to be an overlap of the burning off carbon nanotube material and grafted aminosilane with the higher condensation degree in comparison to the condensed aminosilane molecules burnt off in fraction 1. Fraction 3 of the SII-MWCNTs corresponds to the burning off of highly condensed aminosilane coating. From the comparative 
analysis of all investigated fractions it can be deduced that silanization process improved thermal stability of the MWCNTs sample which is related to the presence of the Si particles at the defect sites. This effect is easily observed when one compares thermal stability of
P-MWCNTs and SII-MWCNTs samples. In this case the oxidation temperature of the MWCNTs after the functionalization performed according to the procedure described in the experiment II increased by $93^{\circ} \mathrm{C}$ (see fractions 4 of P-MWCNTs and SII-MWCNTs).

TABLE

The data obtained from DTG and Voigt fitting of DTG of the investigated samples.

\begin{tabular}{c|c|c|c|c|c|c|c|c}
\hline \hline $\begin{array}{c}\text { Fractions } \\
\text { in DTG }\end{array}$ & \multicolumn{2}{|c|}{ P-MWCNTs } & \multicolumn{2}{c|}{ O-MWCNTs } & \multicolumn{2}{c|}{ SI-MWCNTs } & \multicolumn{2}{c}{ SII-MWCNTs } \\
\hline & Temp. $\left[{ }^{\circ} \mathrm{C}\right]$ & Area [\%] & Temp. $\left[{ }^{\circ} \mathrm{C}\right]$ & Area [\%] & Temp. $\left[{ }^{\circ} \mathrm{C}\right]$ & Area [\%] & Temp. $\left[{ }^{\circ} \mathrm{C}\right]$ & Area [\%] \\
\hline 1 & 380 & 1.52 & - & - & 440 & 13.91 & $257,282,317$ & 19.90 \\
2 & 507 & 33.58 & 489 & 29.84 & 506 & 26.35 & 468 & 33.70 \\
3 & 530 & 30.28 & 515 & 22.64 & 529 & 32.27 & 518 & \\
4 & 550 & 34.62 & 547 & 47.52 & 545 & 27.47 & 643 & 46.40
\end{tabular}

The effects of the silanization routes on the properties of the multiwalled carbon nanotubes clearly show the improvement of its thermal stability along with the increased content of the APTES molecules on the surface. Therefore, from both silanization routes the two-step silanization process is favourable in order to obtain thicker aminosilane layer which preserves the defect sites and makes the system thermally more stable.

\section{Conclusions}

To conclude, the influence of the different silanization procedures on the thermal, structural and vibrational properties of MWCNTs has been presented. The detailed spectroscopic and microscopic analysis of the silanized samples have been shown. Interestingly, it is proved that the silanization process enhances the thermal stability of MWCNTs. This effect is even stronger via the condensation process of the aminosilane molecules around the tubes. In this case the thermal stability of the sample increased by $93^{\circ} \mathrm{C}$ in respect to the starting material.

\section{Acknowledgments}

The authors wish to thank A. Bachmatiuk and A. Jędrzejewska for technical assistance. The work was funded by Polish research granted in 2009-2012.

\section{References}

[1] C. Klumpp, K. Kostarelos, M. Prato, A. Bianco, Biochem. Biophys. Acta 1758, 404 (2006).

[2] Z. Liyun, L. Hongyun, H. Naifei, J. Colloid Interface Sci. 296, 204 (2006).

[3] M. Qingxin, L. Wei, X. Yuehan, Z. Hongyu, L. Zhenwei, Z. Ying, J. Leihua, W. Fang, S. Zhikun, Z. Bin, Y. Bin, J. Phys. Chem. C 112, 3300 (2008).
[4] J. Kathi, K.Y. Rhee, J. Mater. Sci. 43, 33 (2008).

[5] Z.H. Wang, G. Jin, J. Immunol. Meth. 285, 237 (2004).

[6] J. Smits, B. Wincheski, J. Ingram, N. Watkins, J. Jordan, Mater. Res. Soc. Symp. Proc. 739, H7.11.1 (2003).

[7] F. Rusmini, Z. Zhong, J. Feijen, Biomacromolecules 8, 1775 (2007).

[8] L. Valentini, J. Macan, I. Armentano, F. Mengoni, J.M. Kenny, Carbon 44, 2196 (2006).

[9] N. Wong, S. Kam, H. Dai, J. Am. Chem. Soc. 127, $6021(2005)$

[10] Z. Zhou, S. Wang, L. Lu, Y. Zhang, Y. Zhang, Comput. Sci. Technol. 68, 1727 (2008).

[11] L. Vast, G. Philippin, A. Destree, N. Moreau, A. Fonseca, J.B. Nagy, J. Delhalle, Z. Mekhalif, Nanotechnology 15, 781 (2004).

[12] C. Velasco-Santos, A.L. Martinez-Hernandez, M. Lozada-Cassou, A. Alvarez-Castillo, V.M. Castano, Nanotechnology 13, 495 (2002).

[13] M. Yamaura, R.L. Camilo, L.C. Sampaio, M.A. Macedo, M. Nakamura, H.E. Toma, J. Magn. Magn. Mater. 279, 210 (2004).

[14] B. Feng, R.Y. Hong, L.S. Wang, L. Guo, H.Z. Li, J. Ding, Y. Zheng, D.G. Wei, Coll. Surf. A: Physicochem. Eng. Asp. 328, 52 (2008).

[15] J.H.T. Luong, S. Hrapovic, D. Wang, F. Bensebaa, B. Simard, Electroanalysis 16, 132 (2004).

[16] J.H.T. Luong, S. Hrapovic, D. Wang, Electroanalysis 17, 47 (2005)

[17] E. Borowiak-Palen, A. Bachmatiuk, M.H. Rümmeli, T. Gemming, M. Kruszynska, R.J. Kalenczuk, Physica E, Low-Dim. Sys. Nanostruct. 40, 2227 (2008).

[18] W.J. Kim, M.L. Usrey, M.S. Strano, Chem. Mater. 19, 1571 (2007). 
[19] A.C. Dillon, P.A. Parilla, J.L. Alleman, T. Gennett, K.M. Jones, M.J. Heben, Chem. Phys. Lett. 401, 522 (2005).

[20] K.D. Shin, K.W. Ju, I.S. Ji, K.H. Young, L.S. Young, J. Fluorine Chem. 128, 60 (2007).

[21] S. Costa, E. Borowiak-Paleń, M. Kruszyńska, A. Bachmatiuk, R.J. Kaleńczuk, Mater. Sci. Poland 26, 2 (2008)
[22] J. Zhang, H. Zou, Q. Qing, Y. Yang, Q. Li, Z. Liu, X. Guo, Z. Du, J. Phys. Chem. B 107, 3712 (2003).

[23] H.P. Boehm, Advances in Catalysis, Vol. 16, Academic Press, New York 1966, p. 179. 